Research Paper

\title{
Effect of Hemoperfusion Using Polymyxin B-immobilized Fibers on Acute Lung Injury in a Rat Sepsis Model
}

Toshiaki Iba ${ }^{1}$, Isao Nagaoka ${ }^{2}$, Atsushi Yamada ${ }^{1}$, Masataka Nagayama ${ }^{1}$, Takahiro Miki ${ }^{1}{ }^{凶}$

1. Department of Emergency and Disaster Medicine, Juntendo University, Graduate School of Medicine, 2-1-1 Hongo Bunkyo-ku, Tokyo, 113-8421, Japan

2. Department of Host Defense and Biochemical Research, Juntendo University, Graduate School of Medicine, 2-1-1 Hongo Bunkyo-ku, Tokyo, 113-8421, Japan

$\triangle$ Corresponding author: Toshiaki Iba, MD, Professor of Emergency and Disaster Medicine, Juntendo University, 2-1-1 Hongo, Bunkyo-ku Tokyo 113-8421, Japan. E-mail: toshiiba@cf6.so-net.ne.jp; Tel: 81-3-3813-3111 (x 5818); Fax: 81-3-3814-5431

(C) Ivyspring International Publisher. This is an open-access article distributed under the terms of the Creative Commons License (http://creativecommons.org/ licenses/by-nc-nd/3.0/). Reproduction is permitted for personal, noncommercial use, provided that the article is in whole, unmodified, and properly cited.

Received: 2013.03.19; Accepted: 2013.06.09; Published: 2014.01.17

\begin{abstract}
Direct hemoperfusion using polymyxin B-immobilized column (PMX-DHP) is recognized as an effective treatment for septic shock. However, whether its efficacy is limited to cardiovascular dysfunction remains unknown. Therefore, we planned to examine the effects of PMX-DHP in an acute lung injury model. [Materials and methods] Rats were assigned to either PMX-DHP group or control group ( $n=7$ in each). A lung injury was created by the intratracheal instillation of LPS. In PMX-DHP group, an arteriovenous extracorporeal circuit using PMX column was applied for three hours. The same procedure using a dummy column was applied in control group. The lung microcirculation was observed, and adherent leukocytes, $\mathrm{RBC}$ velocity, and the arterial $\mathrm{PaO} 2$ were calculated. Pathological changes and the wet/dry weight ratio of the lungs were examined. [Results] Adherent leukocytes and platelets to the lung venules were recognized at 3 hours, and their numbers increased over time. Treatment with PMX-DHP significantly suppressed these events and helped maintenance of the blood flow and $\mathrm{PaO}_{2}$ levels. The lung edema and the histologic damages were also suppressed. [Conclusions] PMX-DHP improved the microcirculation by suppressing leukocyte and platelet adhesion. PMX-DHP had beneficial effects in a model for acute lung injury.
\end{abstract}

Key words: polymyxin B-immobilized fibers, hemoadsorption, lipopolysaccharide, acute lung injury, intravital microscope

\section{Introduction}

Direct hemoperfusion with polymyxin B-immobilized fibers (PMX-DHP) is a blood purification therapy that was developed to adsorb blood endotoxin in serious infectious disease resulting from infections with Gram-negative bacteria [1]. Thus far, the clinical effect of PMX-DHP is thought to consist of an elevation in the blood pressure (BP) at the time of septic shock [2], and its effects on hemodynamics have been mainly examined in clinical studies. Cruz et al. [3] demonstrated significant improvements in hemodynamics and organ dysfunction and a reduced 28-day mortality rate in patients with severe sepsis and/or septic shock arising from intra-abdominal infections with Gram-negative bacteria (EUPHAS trial).

Another topic is the possible efficacy of this device in subjects with lung injury. Effects on idiopathic pulmonary fibrosis and interstitial pneumonia have been reported [4-6]. Furthermore, Antonelli $\mathrm{M}$ et al. revealed the improvement in $\mathrm{PaO} 2 / \mathrm{FiO} 2$ ratio by using EUPHAS data [7]. These findings suggested that the effects of PMX-DHP are not limited to the improvement of hemodynamics. Therefore, we planned a series of experiments to clarify the effects of 
PMX-DHP on an acute lung injury (ALI) induced by lipopolysaccharide (LPS). In the current experiment, we induced ALI by LPS instillation into trachea, which mimicked pneumonia, the most common cause of ALI.

\section{Methods}

\section{Setup of animal model}

A total of 14 ten-week-old Wistar rats (purchased from Japan Clea Co., Tokyo Japan) were used in the study. All the experimental procedures were conducted after obtaining the approval of the Ethical Committee for Animal Experiments of Juntendo University. All the rats were provided with standard rat chow and water ad libitum. Rats were anesthetized with intraperitoneal sodium thiopental $(100 \mathrm{mg} / \mathrm{kg}$; Pentothal, Sigma Chemical Co., St.Louis, USA). For continuous anesthesia, propofol (Sigma Chemical Co.) infusion and fluids $(10 \mathrm{ml} / \mathrm{kg} / \mathrm{hr}$ sodium chloride $)$ were administered. The rats were tracheotomized, and a sterile metal cannula was inserted into the trachea, followed by mechanical ventilation in the pressure-controlled mode (Servo Ventilator 300; Maquet, Solna, Sweden). The peak inspiratory pressure was 14 $\mathrm{cm} \mathrm{H} 2 \mathrm{O}$, with a positive end-expiratory pressure of 3 $\mathrm{cm} \mathrm{H} 2 \mathrm{O}$. The fractional inspired oxygen concentration (FIO2) was 1.0, the inspiratory: expiratory ratio was $1: 2$, and the respiratory frequency was $30 / \mathrm{min}$. A total of $10.0 \mathrm{mg} / \mathrm{kg}$ of LPS (E. coli O55-B5; Difco Laboratories, Detroit, USA) diluted with $0.15 \mathrm{~mL}$ of sterile physiological saline was instilled via the trachea for one hour.

The animals were assigned to two groups $(n=7$ in each group). In the PMX-DHP group, immobilized PMX-DHP in a miniature column (internal diameter 5 $\mathrm{mm} \times$ length $13 \mathrm{~mm}$, capacity approximately $0.8 \mathrm{~mL}$; amount of immobilized PMX, $3.0 \mathrm{mg}$ ) provided by Toray Medical Co. (Tokyo, Japan) was applied just before the LPS instillation and continued for three hours. In the control group, a dummy column (equal volume but without PMX) was used in the circuit for three hours. Above surgical maneuver was undergone under the sterile condition, and no antibiotic agent was used throughout the experiment.

\section{Microscopic examination}

For the intra-vital microscopic examination, a thoracosternotomy was performed at the level of the fifth intercostals space under anesthesia, and an area approximately $10 \mathrm{~mm}$ in diameter of the left lower lobe was fixed to a glass slide and immobilized on a special stand. The microcirculation was then observed using the microscopic system (Nikon Microphot-FX Microscope, Nikon Co., Ltd., Tokyo, Japan) at 3 and 6 $\mathrm{hr}$ after LPS instillation. Each field was recorded for 30 seconds at 30 frames/sec using a video system (Ikegami CA-300, Ikegami Tsushinki Co., Ltd., Tokyo, Japan).

At the end of the experiment, mercy killing was performed, and the right lung was excised. Then the upper lobes were fixed in $10 \%$ buffered formalin, embedded in paraffin, stained with hematoxylin/eosin (H-E), and examined using light microscopy. The middle lobes were served for the measurement of wet/dry weight ratio.

\section{Measurements and observations}

In each animal, six successive fields were randomly selected and the images obtained were used to document and analyze the findings. A leukocyte was defined as adherent to venule if it remained stationary for 30 seconds. The numbers of adherent leukocytes in the pulmonary venules at 3 and 6 hours after LPS instillation were counted by two independent persons and the mean value was calculated.

To analyze the RBC velocity, the venule was observed and the images were recorded using a high-speed camera (Memrecam GX-1, Nac Image Technology Inc., Tokyo, Japan) at 3 and 6 hours after LPS instillation. Then, the RBC velocity was calculated by particle image velocimetry (Digimo Co., Ltd., Tokyo, Japan) [8].

Blood samples were analyzed by blood gas analyzer (Radiometer BMS Mk3, Copenhagen, Denmark). To measure the partial pressure of oxygen $(\mathrm{PaO} 2)$, blood samples were obtained from the catheter placed in the femoral artery at 3 and 6 hours after LPS instillation.

The lung damage was evaluated by semi-quantitative method. The degree of thrombus formation, blood cells infiltration in interstitial space, blood cells infiltration to alveolus and parenchymal destruction was evaluated as mild, moderate or severe and the total score was calculated.

Lung wet/dry weight ratio was determined by drying (at $100{ }^{\circ} \mathrm{C}$ for 48 hours) and weighing the right middle lobe.

\section{Statistical analysis}

All the data are expressed as the mean \pm standard deviation. A statistical analysis was performed using the Mann-Whitney $U$ test to compare the mean values between the two groups using the Stat View II statistical software package for Macintosh. Differences were deemed statistically significant when the $P$ value was less than 0.05 .

\section{Results}

At three hours after LPS instillation, leukocyte adhesion to the endothelial surface of the venule was 
observed in both the control and the PMX-DHP groups. In addition to leukocytes, platelets had stuck to the endothelium and leukocytes in the control group, whereas, minimal sticking was observed in the PMX-DHP group (Fig. 1A). These changes increased over time, and the lining consisted of adherent leukocytes and platelets covering the surface. As a result, the vascular lumen became significantly narrow at six hours in the control group. Although the number of leukocytes increased, the lining was not observed in the PMX-DHP group and the blood flow was better maintained (Fig. 1B).

A low magnified view revealed cloudy alveoli with exudates in the control group (Fig. 2A), increasing over time. Meanwhile, the alveoli remained clear in the PMX-DHP group, even at 6 hours (Fig. 2B).

The pathological findings in H-E stained specimens were comparable to that of the intravital microscopic examination. Widespread neutrophil and platelet plugging in the capillary, infiltration to interstitial and intra-alveolar space and destruction of the lung parenchyma was observed in the control group, and these changes were kept less in the PMX-DHP group (Fig. 2C). The degree of the histological damage calculated by semi-quantitative method was significantly suppressed in PMX-DHP group (3.57 \pm 1.90 )
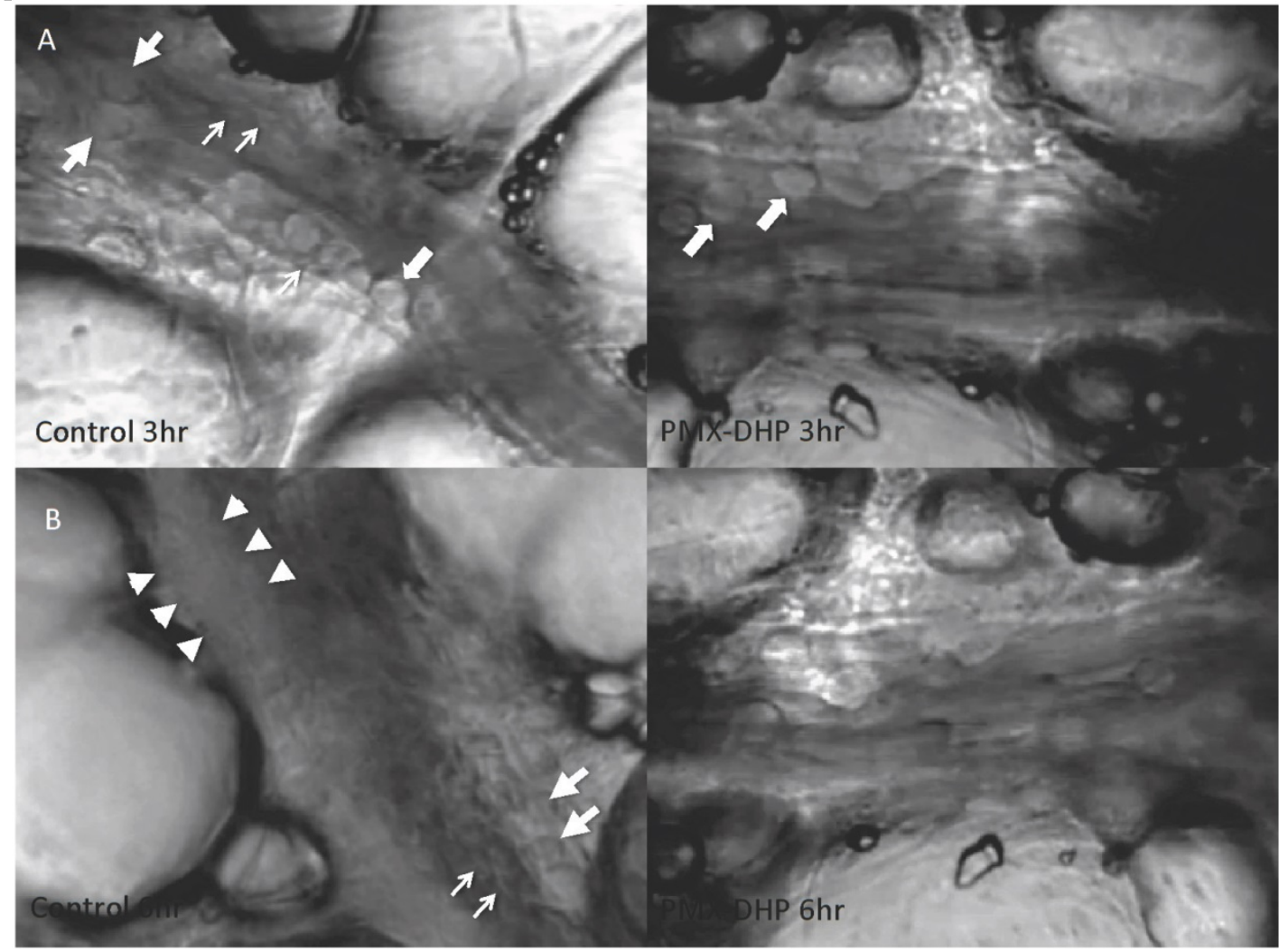

Fig. I. Status of lung microcirculation at 3 and 6 hours after LPS inhalation. At 3 hours (row A), in addition to the adherent leukocytes (large arrows), platelets had stuck to the endothelium and leukocytes (small arrows) in the control group (left). Leukocytes, but very few platelets, adhered to the endothelium in the PMX-DHP group. (Objective lens with a magnifying power of $\times 20$ ). At 6 hours (row B), a thick lining formed from adherent leukocytes, platelets and fibrin deposition (between the arrowheads) had narrowed the vessel cavity in the control group. The large arrow indicates a leukocyte, and the small arrows indicate platelets. Blood flow was maintained in the PMX-DHP group. (Objective lens with a magnifying power of $\times 20$ ) compared to the control group $(7.29 \pm 2.36, P<0.01)$ (Table 1).

Table I. Histological examination of the lung.

\begin{tabular}{|c|c|c|c|c|c|c|}
\hline animal No. & & $\begin{array}{l}\text { thrombus in } \\
\text { vessel }\end{array}$ & $\begin{array}{l}\text { blood cell } \\
\text { infiltration } \\
\text { to intersti- } \\
\text { tial space }\end{array}$ & $\begin{array}{l}\text { blood cell } \\
\text { infiltra- } \\
\text { tion to } \\
\text { alveolus }\end{array}$ & $\begin{array}{l}\text { paren- } \\
\text { chymal } \\
\text { destruc- } \\
\text { tion }\end{array}$ & total score \\
\hline \multirow[t]{8}{*}{ Control } & 1 & +++ & ++ & +++ & +++ & 11 \\
\hline & 2 & + & + & ++ & ++ & 6 \\
\hline & 3 & ++ & ++ & + & + & 6 \\
\hline & 4 & + & + & ++ & + & 5 \\
\hline & 5 & ++ & + & ++ & & 5 \\
\hline & 6 & + & ++ & +++ & +++ & 9 \\
\hline & \multirow[t]{2}{*}{7} & ++ & ++ & +++ & ++ & 9 \\
\hline & & & & \multicolumn{2}{|c|}{ mean \pm STD } & $7.29 \pm 2.36$ \\
\hline \multirow[t]{8}{*}{ PMX-DHP } & 1 & ++ & + & ++ & + & 6 \\
\hline & 2 & + & & + & & 2 \\
\hline & 3 & + & + & + & & 3 \\
\hline & 4 & ++ & + & ++ & + & 6 \\
\hline & 5 & + & & + & + & 3 \\
\hline & 6 & + & + & + & + & 4 \\
\hline & 7 & + & & & & 1 \\
\hline & & & & \multicolumn{2}{|c|}{ mean \pm STD } & $3.57 \pm 1.90$ \\
\hline
\end{tabular}

PMX-DHP: direct hem

(+: mild, ++: moderate, +++: severe)

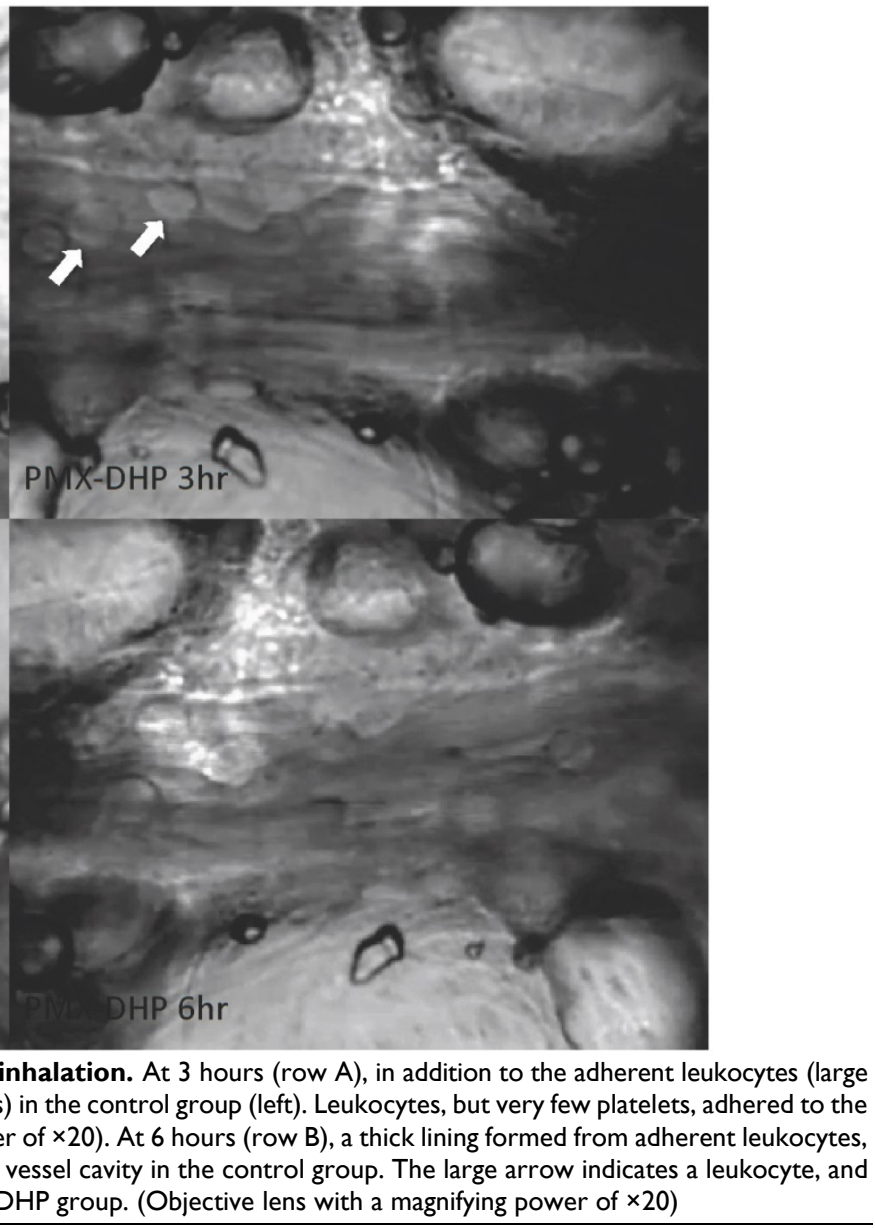



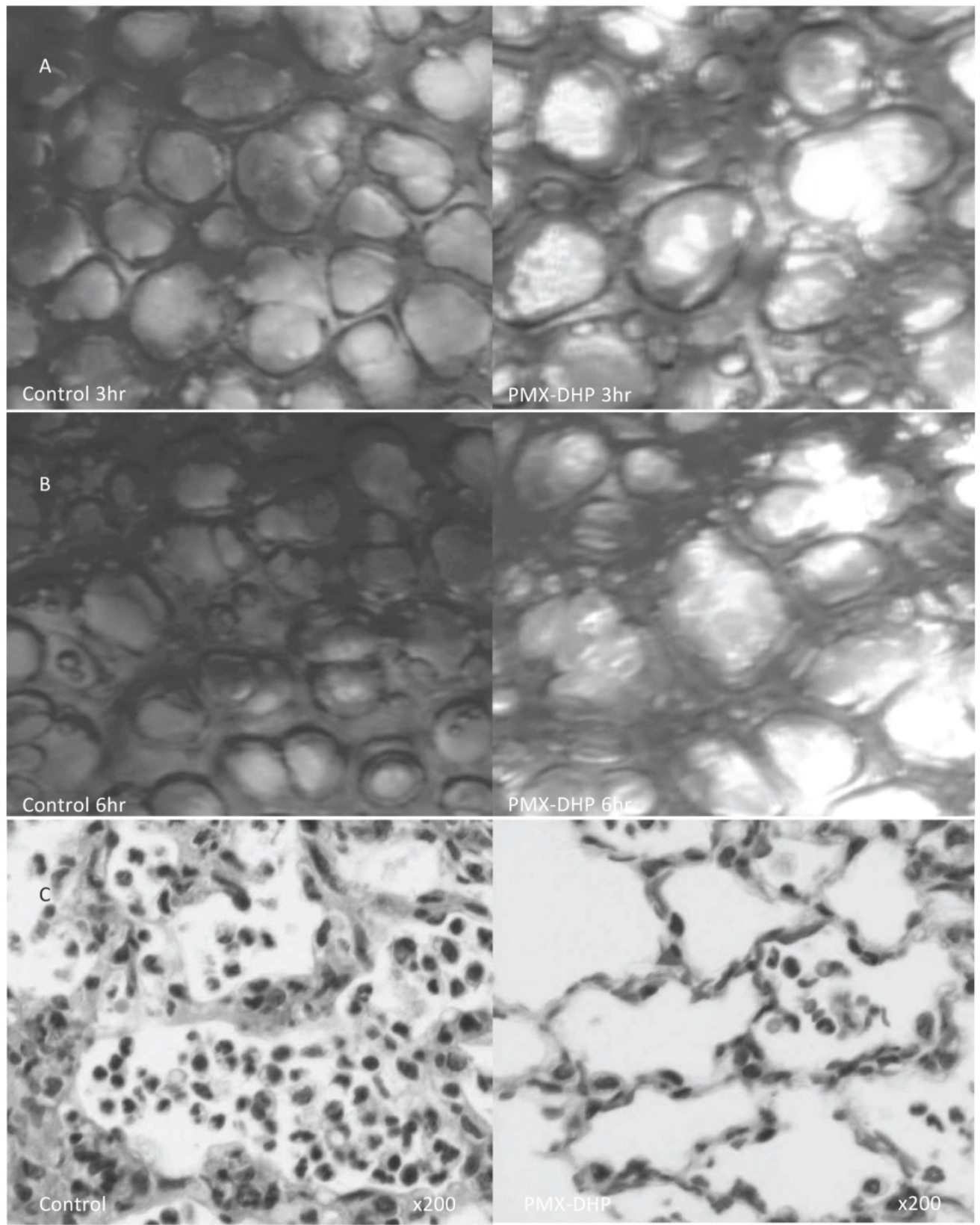

Fig. 2. Status of lung alveoli at 3 and 6 hours and the pathologic finding at $\mathbf{6 h r}$ after LPS inhalation. At 3 hours (row A), cloudy alveoli with exudates were observed under a low magnification in the control group. The alveoli were clear in the PMX-DHP group. At 6 hours (row B), the translucency of the alveoli had further decreased in the control group. (Objective lens with a magnifying power of $\times 4$ ). Pathologic changes in hematoxylin/eosin stained specimen (row C) was consistent with acute lung injury in the control group, including widespread neutrophil infiltration with interstitial and intra-alveolar pulmonary edema and destruction of the normal lung parenchyma in the control group. While, these changes were less significant in PMX-DHP group. (original magnification $\times 200$ )

An off-line video analysis revealed that the number of adherent leukocytes increased after LPS instillation. This increase was significantly suppressed in the PMX-DHP group ( $P<0.05$ at 6 hours) (Fig. 3, left). The RBC velocity decreased over time, with values of $1.51 \pm 0.54 \mathrm{~mm} / \mathrm{sec}$ observed at 3 hours and $0.83 \pm 0.40 \mathrm{~mm} / \mathrm{sec}$ observed at 6 hours in the control group. The RBC velocity was better maintained in the PMX-DHP group, and the difference was significant at 6 hours $(P<0.05)$ (Fig. 3 , middle). No difference in the mean $\mathrm{PaO} 2 / \mathrm{FIO} 2$ ratio was observed between the groups at 3 hours, with a value of around $300 \mathrm{mmHg}$ observed in both groups. However, the ratio decreased steeply to $152.9 \pm 48.2 \mathrm{mmHg}$ at 6 hours in the control group, while the value decreased to $218.6 \pm$ $54.6 \mathrm{mmHg}$ in the PMX-DHP group (Fig. 3, right).

Wet/dry weight ratio of the right middle lobe of PMX-DHP group (5.58 \pm 0.80$)$ was significantly lower compared with the control group $(6.54 \pm 0.87, P<$ $0.05)$. 

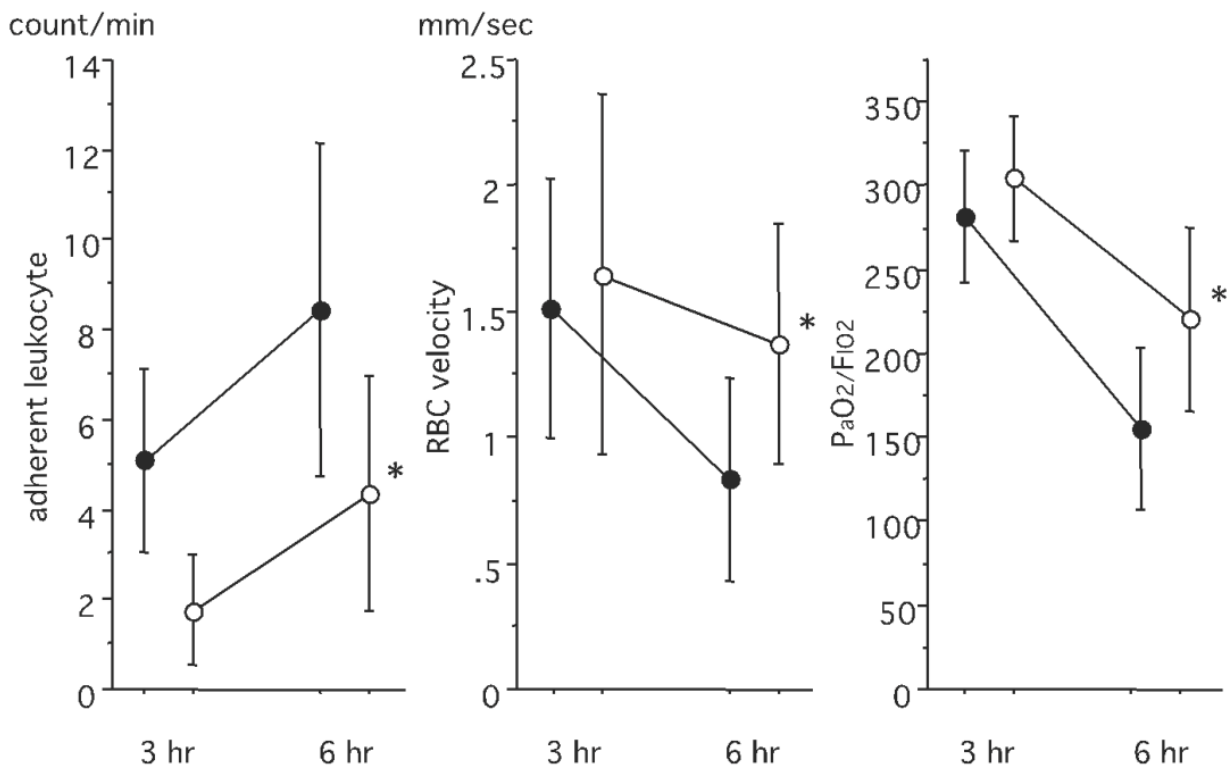

Fig. 3. Comparison of leukocyte adhesion, $\mathbf{R B C}$ velocity and $\mathbf{P a O}_{2}$ levels. The number of adherent leukocytes was higher in the control group, and the difference was significant at 6 hours. RBC velocity was better maintained in the PMX-DHP group, and the difference was significant at 6 hours. $\mathrm{PaO}_{2} / \mathrm{FIO}_{2}$ decreased over time, and the difference was significant between 3 hours and 6 hours in both groups $(P<0.05$ respectively, $\mathrm{n}=7$ in each group). $\mathrm{PaO}_{2} / \mathrm{FIO}_{2}$ was better maintained in the PMX/DHP group. ( $\bullet$ : control group, ०: PMX-DHP group; each symbol expresses the mean and standard deviation; $* P<0.05$, compared with the control group).

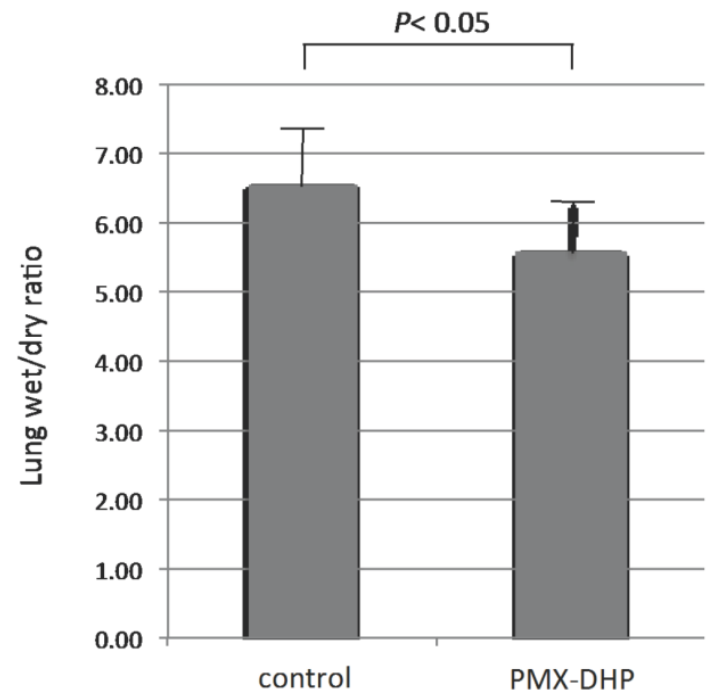

Fig. 4. Comparison of wet/dry lung weight ratio. Wet/dry weight ratio of the right middle lobe was significantly higher in the control group compared with the PMX-DHP group $(P<0.05)(n=7$ in each group). Data are expressed as the mean and standard deviation.

\section{Discussion}

Although PMX-DHP is a popular treatment for septic shock in Japan, only a few well-designed randomized controlled studies have been performed [9]. First, Vincent et al. [10] reported the results of an open-label pilot study. In their study, sepsis patients with intra-abdominal infection were randomly assigned to either a PMX-DHP or control group, and PMX-DHP revealed a significant improvement in the cardiac index (CI), the left ventricular stroke index
(LVSWI), and the oxygen delivery index $\left(\mathrm{DO}_{2} \mathrm{I}\right)$. These observations were further confirmed by a large-scale study [3]. Based on these reports, the mechanism of action of PMX-DHP is thought to be an improvement in the hemodynamic status at the time of septic shock. However, recent clinical studies have indicated potential effects of PMX-DHP besides those influencing cardiovascular dysfunction. For example, beneficial effects have been reported in patients with idiopathic pulmonary fibrosis [4], interstitial pneumonia [5], and acute respiratory distress syndrome [11]. Takahashi et al. [12] demonstrated the significant improvement in $\mathrm{PaO} 2 / \mathrm{FIO} 2$ ratio following the decrease of S100A12, an extracellular newly identified RAGE-binding protein, and endotoxin after PMX-DHP in post-surgical septic patients. These findings raise the question of whether PMX-DHP has beneficial effects in patients with ALI. To address this question, we created a rat model of septic ALI and examined the effects of PMX-DHP.

ALI is characterized by severe alveolar and interstitial edema with a large amount of neutrophil infiltration, leading to increased vascular permeability and impaired gas exchange $[13,14]$. We previously reported that neutrophils play key roles in interstitial edema, alveolar injury and microcirculatory disturbance [15]. In the present model, the pathologic findings revealed the consistent changes with ALI in the control group, including widespread leukocyte infiltration with interstitial and intra-alveolar pulmonary edema and destruction of the normal lung parenchyma. And, the serial observation under the intravi- 
tal microscopy demonstrated the first changes observed examination were an increase in the adherence of leukocytes followed by platelet sticking, leading to the formation of a lining on the endothelium. Then, the narrowing of the vascular lumen resulted in a decrease in blood flow through the pulmonary microcirculation. In a previous study, we demonstrated that these events lead to alveolar damage and interstitial edema $[15,16]$. Alveolar and interstitial edema, visualized as a reduction in translucency, were also recognized in the present model, and such damage was effectively reduced by the PMX-DHP treatment. However, the changes, including increased adherent leukocytes, decreased blood flow and increased interstitial edema were not significant between the groups at 3 hours. It became obvious at 6 hours in the control group and lead to the disturbance of gas exchange. While, the histological changes and $\mathrm{PaO} 2 / \mathrm{FIO} 2$ ratio was maintained better in PMX/DHP group. The lower wet/dry ratio in PMX-DHP group further confirmed the effect.

According to the mechanism of action of PMX-DHP, we focused on the interaction between the blood cells and vascular endothelium in this study. Activated leukocytes are known to play major roles in the development of organ dysfunction during sepsis [16]. So, the trapping of leukocytes in the column seems reasonable $[17,18]$. Interestingly, Kumagai et al. [19] reported that PMX-DHP selectively removes activated neutrophils and thus, reduces the ability of circulating cells to cause endothelial damage. Their flow cytometric analysis indicated that activated neutrophils with high levels of CD11b/CD64 expression and low levels of CXCR1/CXCR2 expression showed preferential adhesion to PMX filters. They also reported that neutrophils isolated from the blood after ex vivo PMX perfusion caused less damage to an endothelial cell monolayer. The type of adherent leukocyte could not be identified by the intravital microscopy, however, we speculate the major part was neutrophils since the pathological changes in H-E stain demonstrated the neutrophil invasion to the alveoli. Tsushima et al. [20] reported that neutrophil count in bronchoalveolar lavage fluid was significantly decreased with the treatment of PMX-DHP in hydrochloric acid-induced lung injury model of rat. In addition to the changes in the cell counts, we have previously reported the decrease in proinflammatory cytokine levels and the improvement in survival [16]. Other than those, Kushi et al. [21] demonstrated a decrease in the neutrophil elastase level, and Naka [22] showed a decrease in oxygen radical species after PMX-DHP treatment. These mechanisms may contribute to minimizing the lung injury.

Except leukocyte trapping, our findings sug- gested that the effects on platelet may also play a role. During PMX-DHP, the decrease of the platelet count is often recognized and this decrease is generally thought to be unfavorable [23]. However, since the positive effect of the elimination of the activated platelets was demonstrated in this study, we think that we should not be afraid of this phenomenon. Indeed, the roles for platelets in inflammation have attracted much attention recently. Platelets have been shown to interact with microorganismus and they are known to release inflammatory factors including interleukin (IL)-1, P-selectin, and CD40 ligand (CD40L) [24]. Platelets also express immune-related receptors, such as toll-like receptors (TLRs) [25]. TLR2 and 4 recognize pathogen-associated molecular patterns (PAMPs) and promotes the formation of platelet-neutrophil aggregates leading to the neutrophil extracellular traps (NETs) [26]. These structures further bind to platelets and red blood cells and form thrombi optimized as lining in the venule in this experiment. As a result, the decrease of the platelet count in the peripheral blood has been known to correlates to the severity of sepsis [27]. As mentioned before, the decrease of the platelet counts during PMX-DHP does not reflect the worsening of the condition. We think the decreased platelet count rather expresses the therapeutic effect. Other than the count changes, Kase et al. [28] performed a comparative study and reported that circulating 2-AG from platelets decreased after treatment, leading to an improvement in the hemodynamic state. These mechanisms, such as the trapping of activated platelets and the adsorption of 2-AG, have also attracted attention as a breakthrough in the action mechanism.

\section{Conclusion}

PMX-DHP improved lung damage in an ALI model. PMX-DHP leads the maintenance of lung microcirculation, vascular permeability and oxygenation. Although the mechanism of action of this treatment was not fully elucidated, the suppression of the adherence of leukocytes and platelets might play roles. Further examination in the clinical study is warranted to verify these effects.

\section{Abbreviations}

PMX-DHP: polymyxin B-immobilized column; BP: blood pressure; ALI: acute lung injury; LPS: lipopolysaccharide; FIO2: fractional inspired oxygen concentration; H-E: hematoxylin/eosin; PaO2: partial pressure of oxygen; CI: cardiac index; LVSWI: left ventricular stroke index; $\mathrm{DO}_{2} \mathrm{I}$ : oxygen delivery index; IL: interleukin; CD40L: CD40 ligand; TLRs: toll-like receptors; PAMP: pathogen-associated molecular pattern; NETs: neutrophil extracellular traps. 


\section{Acknowledgements}

A part of this study was presented at the 28th International Symposium on Intensive Care and Emergency Medicine. This work was financially supported by the National Science and Technology Supporting Program (20590456).

\section{Competing Interests}

The authors have declared that no competing interest exists.

\section{References}

1. Shoji $\mathrm{H}, \mathrm{Tani} \mathrm{T}$, Hanasawa $\mathrm{K}$, et al. Extracorporeal endotoxin removal by polymyxin $\mathrm{B}$ immobilized fiber cartridge designing and antiendotoxin efficacy in the clinical application. Ther Apher. 1998; 2: 3-12.

2. Shimizu T, Hanasawa K, Sato K, et al. Direct hemoperfusion with polymyxin-B-immobilized fiber columns improves septic hypotension and reduces inflammatory mediators in septic patients with colorectal perforation. Langenbecks Arch Surg. 2008; 394: 303-11.

3. Cruz DN, Antonelli M, Fumagalli R, et al. Early use of polymyxin B hemoperfusion in abdominal septic shock: the EUPHAS randomized controlled trial. JAMA. 2009; 301: 2445-52.

4. Tachibana K, Inoue $Y$, Nishiyama A, et al. Polymyxin-B hemoperfusion for acute exacerbation of idiopathic pulmonary fibrosis: serum IL-7 as a prognostic marker. Sarcoidosis Vasc Diffuse Lung Dis. 2011; 28: 113-22.

5. Enomoto N, Suda T, Uto T, et al. Possible therapeutic effect of direct haemoperfusion with a polymyxin B immobilized fibre column (PMX-DHP) on pulmonary oxygenation in acute exacerbations of interstitial pneumonia. Respirology. 2008; 13: 452-60.

6. Hara S, Ishimoto H, Sakamoto N, et al: Direct hemoperfusion using immobilized polymyxin B in patients with rapidly progressive interstitial pneumonias: a retrospective study. Respiration. 2011; 81: 107-17.

7. Antonelli M, Fumagalli R, Cruz DN, et al. PMX endotoxin removal in the clinical practice: results from the EUPHAS trial. Contrib Nephrol. 2010; 167: 83-90.

8. Iba T, Kidokoro A, Fukunaga M, et al. Factor Xa-inhibitor (DX-9065a) modulates the leukocyte-endothelial cell interaction in endotoxemic rat. Shock. 2002; 17: 159-62.

9. Cruz DN, Perazella PA, Bellomo R, et al. Effectiveness of polymyxin B-immobilized fiber column in sepsis: a systematic review. Crit Care. 2007; 11: R47-58.

10. Vincent JL, Laterre PF, Cohen J, et al. A pilot-controlled study of a polymyxin B-immobilized hemoperfusion cartridge in patients with severe sepsis secondary to intra-abdominal infection. Shock. 2005; 23: 400-5.

11. Nakamura T, Fujiwara N, Sato E, et al. Effect of polymyxin B-immobilized fiber hemoperfusion on serum high mobility group box-1 protein levels and oxidative stress in patients with acute respiratory distress syndrome. ASAIO J. 2009; 55: 395-9.

12. Takahashi G, Hoshikawa K, Matsumoto N, et al. Changes in serum S100A12 and sRAGE associated with improvement of the $\mathrm{PaO}(2) / \mathrm{FiO}(2)$ ratio following PMX-DHP therapy for postoperative septic shock. Eur Surg Res. 2011; 47: $135-40$.

13. Weiner-Kronish JP, Gropper MA, Matthay MA. The adult respiratory distress syndrome: definition and prognosis, pathogenesis and treatment. Br J Anaesthesia. 1990; 65: 107-29.

14. Havemann K, Gramse G. Physiology and pathophysiology of neutral proteinases of human granulocytes. Adv Exp Med Biol. 1984; 84: 1-20.

15. Iba T, Kidokoro A, Fukunaga M, et al. Pretreatment of sivelestat sodium hydrate improves the lung microcirculation and alveolar damage in lipopolysaccharide-induced acute lung inflammation in hamsters. Shock. 2006; 26: 95-8.

16. Iba T, Okamoto K, Kawasaki S, et al. Effect of hemoperfusion using polymyxin B-immobilized fibers on non-shock rat sepsis model. J Surg Res. 2011; 171: 755-61.

17. Yoshida T, Kodama M, Tamura Y, et al. Direct hemoperfusion with a polymyxin B-immobilized fiber column eliminates neutrophils and improves pulmonary oxygenation--a comparison of two cases with acute exacerbation of idiopathic pulmonary fibrosis. J Jpn Resp Soc. 2007; 45: 890-7.

18. Abe S, Seo $\mathrm{Y}$, Hayashi $\mathrm{H}$, et al. Neutrophil adsorption by polymyxin B-immobilized fiber column for acute exacerbation in patients with interstitial pneumonia: a pilot study. Blood Purif. 2010; 29: 321-6.

19. Kumagai T, Takeyama N, Yabuki T, et al. Apheresis of activated leukocytes with an immobilized polymyxin B filter in patients with septic shock. Shock. 2010; 34: 461-6.

20. Tsushima K, Koizumi T, Yoshikawa S, et al. Polymyxin B immobilized column is effective for hydrochloric acid-induced lung injury in rats. Eur J Pharmacol. 2006; 535: 270-9.
21. Kushi H, Miki T, Nakahara J, et al. Hemoperfusion with an immobilized polymyxin B column reduces the blood level of neutrophil elastase. Blood Purif. 2006; 24: 212-7.

22. Naka T, Shinozaki M, Akizawa T, et al. The effect of continuous veno-venous hemofiltration or direct hemoperfusion with polymyxin B-immobilized fiber on neutrophil respiratory oxidative burst in patients with sepsis and septic shock. Ther Apher Dial. 2006; 10: 7-11.

23. Ikeda T. Hemoadsorption in critical care. Ther Apher. 2002, 6:189-92.

24. Fitzgerald JR, Foster TJ, Cox D. The interaction of bacterial pathogens with platelets. Nat Rev Microbiol. 2006; 4: 445-57.

25. Blair P, Rex S, Vitseva O, et al. Stimulation of Toll-like receptor 2 in human platelets induces a thromboinflammatory response through activation of phosphoinositide 3-kinase. Circ Res. 2009; 104: 346-54.

26. Clark SR, Ma AC, Tavener SA, et al. Platelet TLR4 activates neutrophil extracellular traps to ensnare bacteria in septic blood. Nat Med. 2007; 13: 463-9.

27. Iba T, Gando S, Murata A, et al. Predicting the severity of systemic inflammatory response syndrome (SIRS)-associated coagulopathy with hemostatic molecular markers and vascular endothelial injury markers. J Trauma. 2007; 63:1093-8.

28. Kase Y, Obata T, Okamoto Y, et al. Removal of 2-AG by direct hemoperfusion therapy with polymyxin B immobilized fibers benefits patients with septic shock. Ther Apher Dial. 2008; 12: 374-80. 